\title{
Functionalized Surfaces to Improve Imaging Conditions in Liquid Cell Transmission Electron Microscopy
}

\author{
John M. Miller ${ }^{1}$, Daan Hein Alsem ${ }^{2}$, Norman Salmon ${ }^{2}$, Nils E. Johnson ${ }^{3}$, and James E. Hutchison ${ }^{1,3}$ \\ ${ }^{1}$ Dune Sciences, Eugene, OR, USA \\ ${ }^{2}$ Hummingbird Scientific, Lacey, WA, USA \\ ${ }^{3}$ University of Oregon, Department of Chemistry and Biochemistry, Eugene, OR, USA
}

Despite the substantial inherent interest in high resolution imaging of liquid/solid material interfaces and biological structures, many traditional characterization techniques simply do not have the necessary spatial resolution. TEM, however, is truly a keystone technique in chemical, material, biomedical, and nanoscience, because of its unique ability to obtain nanoscale imaging resolution of materials' internal structures and changes after a reaction. More recently, the use of TEM has moved beyond the traditional post-mortem imaging in vacuum and has also focused on in-situ experiments inside the TEM in liquid environments [1-6]. All these studies use a variation of the concept of stacking of two microfabricated chips with electron transparent membranes together and sealing it from the vacuum inside the TEM.

In liquid cell TEM, the sample is suspended in a liquid environment that is sandwiched between the two chips in a relatively thin layer (typically 100's of $\mu \mathrm{m}$ ). While this creates the opportunity to observe the behavior of one's sample in the liquid medium, this configuration can also create challenges when imaging at high resolution due to the greater mobility of the sample when compared to imaging under vacuum. When imaging nanoparticles, this can be especially challenging due to the relatively small viewing area, particularly at high magnification, and the propensity of the sample being imaged to wander out of the frame. Further, while the liquid layer is relatively thin, 100-500 $\mu \mathrm{m}$, the depth of focus required to image particles across this layer is not practical for most TEM instruments. One strategy to address this challenge is to tether or immobilize the sample onto the substrate membrane using surface functionalization (See fig 1). This approach localizes the sample on the membrane to improve image focus and resolution for both static and dynamic liquid experiments. Further, the appropriate selection of surface functionalization can simplify sample preparation protocols, improve the dispersion and uniformity of the sample, and minimize unwanted artefacts associated with sample preparation.

In this collaborative effort between the University of Oregon, Dune Sciences and Hummingbird Scientific, we investigate the role that surface functionalization of the electron transparent membrane plays in imaging nanoparticles under different imaging conditions. Specifically, using Hummingbird Scientific's latest liquid cell TEM technology combined with SMART Grids surface functionalization by Dune Sciences, we study imaging conditions of two different ligand-stabilized Au nanoparticles in liquid environments as well as in air. In the first example, $1.5 \mathrm{~nm}$ mercaptoethanesulfonate (MES) stabilized gold nanoparticles were self-assembled onto amine-functionalized membranes through electrostatic interactions. The MES group bears a net negative charge that is attracted to the positively charged amine surface. The second system utilized $1.5 \mathrm{~nm}$ thiocholine stabilized Au nanoparticles self-assembled onto one membrane that had been functionalized with carboxylate surface chemistry. The thiocholine ligand stabilizer has a permanent positive charge that electrostatically binds to negative surface charge of the carboxylate. For both systems, we compare STEM imaging of the self-assembled arrays under both static and flowing liquid conditions, in addition to in air (sealed cell, but with no water) and in vacuum. We image at different magnifications, different beam currents, and different 
imaging dwell times for each of these environments to characterize the behavior of the nanoparticles. Depending on the imaging conditions, we observe a number of primary and secondary effects include beam-induced artifacts, nanoparticle growth, and surface migration of the nanoparticles. Further, we observed different effects depending on the surface functionalization/nanoparticle interactions. Figure 2 shows one such example of the MES-stabilized nanoparticles and beam-induced growth that occurred while imaging in STEM mode at $225 \mathrm{kX}$. There are, however, imaging conditions in which these artifacts can be minimized to provide valuable insight to the dynamic behavior of samples in solution. In this presentation, we report the results of these studies and lessons learned for imaging nanoparticles.

References:

[1] MJ Williamson, RM Tromp, PM Vereecken, R Hull, and FM Ross, Nature Materials 2 (2003), p. 532.

[2] H. Zheng, RK Smith, YW Jun, C Kisielowski, U Dahmen, and AP Alivisatos, Science 324 (2009), p. 1309.

[4] JE Evans, KL Jungjohann, ND Browning, and I Arslan, Nano Letters 11, (2011),p. 2809.

[5] D Li, MH Nielsen, JRI Lee, C Frandsen, JF Banfield, and JJ De Yoreo, Science 336 (2012), p. 1014.

[6] TT Woehl, KL Jungjohann, JE Evans, I Arslan, WD Ristenpart, and ND Browning, Ultramicroscopy 127 (2013), p. 53.

Sample chips with

nitride membrane

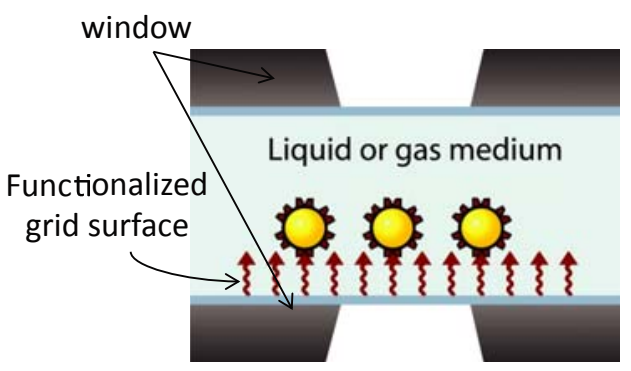

Figure 1: Liquid cell geometry showing a functionalized membrane and nanoparticles assembled on the surface functionalization.
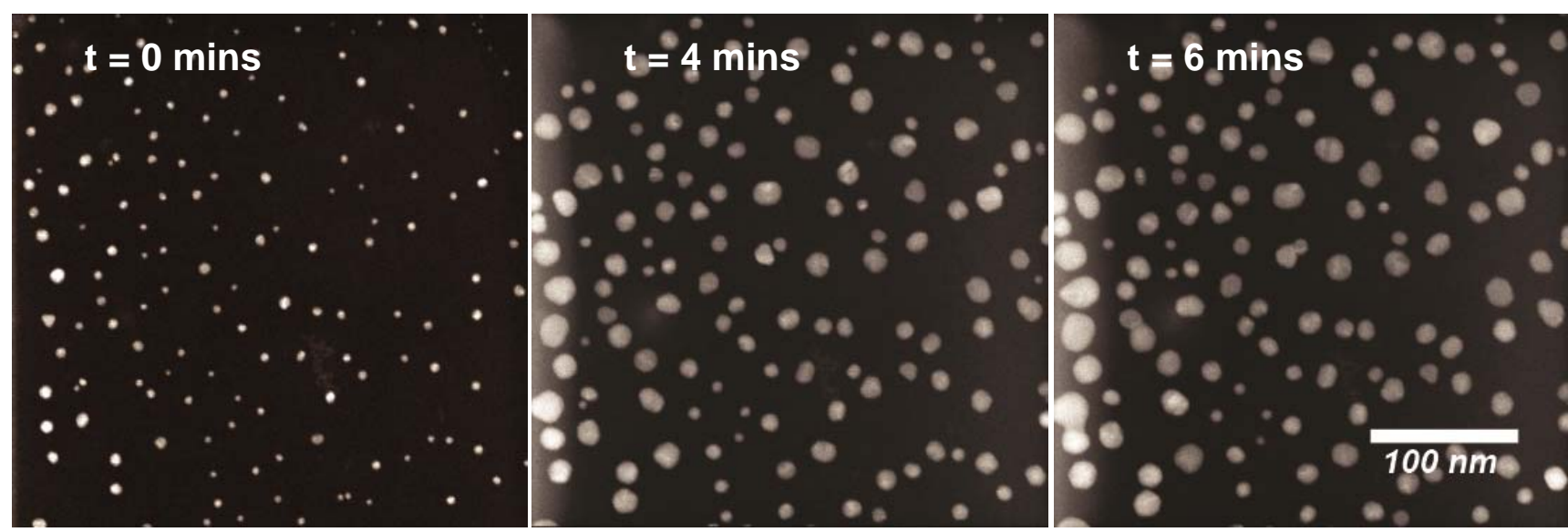

Figure 2. STEM images of $1.5 \mathrm{~nm}$ Au nanoparticles stabilized with mercaptoethanesulfonate assembled on amine-functionalized membranes using in the liquid cell holder. Images taken at $225 \mathrm{Kx}$ using Titan $300 \mathrm{keV}$ TEM with STEM detector at the exposure times shown. Significant particle growth was observed due to reorganization of Au NPs on the membrane surface. 